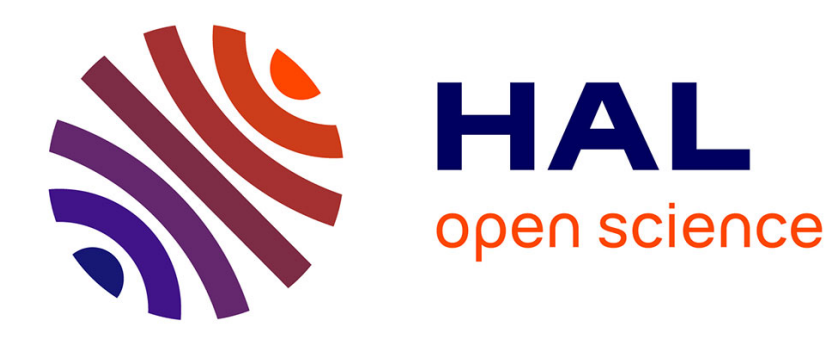

\title{
Le theatrum mundi de Michel Vinaver
}

Marie-Hélène Boblet

\section{To cite this version:}

Marie-Hélène Boblet. Le theatrum mundi de Michel Vinaver. Revue Esprit, 2009, 358, pp.31-50. halshs-00461858

\section{HAL Id: halshs-00461858 \\ https://shs.hal.science/halshs-00461858}

Submitted on 6 Mar 2010

HAL is a multi-disciplinary open access archive for the deposit and dissemination of scientific research documents, whether they are published or not. The documents may come from teaching and research institutions in France or abroad, or from public or private research centers.
L'archive ouverte pluridisciplinaire HAL, est destinée au dépôt et à la diffusion de documents scientifiques de niveau recherche, publiés ou non, émanant des établissements d'enseignement et de recherche français ou étrangers, des laboratoires publics ou privés. 


\section{Le theatrum mundi de Michel Vinaver}

Michel Vinaver, dont l'ultime pièce, le livret 11 Septembre 2001 avait été créée au Théâtre de la Colline en juin 2006, est un auteur comblé. Début 2008 étaient simultanément à l'affiche 11 Septembre 2001, Les Coréens, Par-dessus Bord. Ecrite entre 1967 et 1969, montée au printemps pour la première fois dans son intégralité par Christian Schiaretti pour le TNP de Villeurbanne, cette pièce dure sept heures exultantes et passionnantes. Elles ressuscitent le tournant du capitalisme paternaliste au libéralisme sauvage, et illustrent la religion du marketing par la promotion de Bleu-Blanc-Rouge, papier hygiénique de luxe qui doit permettre au produit français de résister à la concurrence américaine. Jusqu'où peut aller le protectionnisme national... Enfin, entrée en janvier 2009 au répertoire de la Comédie française, L'Ordinaire - et plusieurs autres pièces de Michel Vinaver- était comme appelé par l'actualité. Le Théâtre de La Commune, dans le cycle «L'Idéal L'Utopie », présentait simultanément King mis en scène par Arnaud Meunier. La Colline monta en juin Nina c'est autre chose, exemple d'un théâtre de chambre plus intime. Après que son théâtre eut connu dans les années 70 la faveur des grands (Planchon, Serreau, Vitez, Lassalle, Didym...), il y eut des années sans Vinaver. Le voilà donc de retour, comme si les déflagrations du début du vingt-et-unième siècle rappelaient aux metteurs en scène la pertinence d'une œuvre attachée à faire voir et entendre ce réel imprégné des traces mentales de mai 68 et des suites économiques de la première crise pétrolière et économique : «Mai 68 se fait contre le capitalisme, contre le marketing, contre l'asservissement de l'humanité ; or le libéralisme économique a trouvé un nouvel élan, a fait preuve d'une capacité à se régénérer parce qu'il est capable de rejeter ses déchets : thèmes de l'expulsion, de l'excrément... On en revient étonnamment à Par-dessus bord, non ? ${ }^{1}$

\section{Par-dessus bord ou l'euphorie de l'âge homérique}

Le succès actuel de ce théâtre est relatif aux sujets qui l'occupent: le développement exponentiel du capitalisme et la course effrénée à la consommation, la logique de la prédation

\footnotetext{
${ }^{1}$ M. Vinaver, entretien avec Marie-José Sirach, L'Humanité, 13 mai 2008.
} 
dans les relations humaines et la désolation solitaire d'individus monadiques qu'aucune communauté, à moins de radicalisation ethnocentrique, ne rattache plus les uns aux autres. «Rien ne se relie plus à rien, et tout est lié », comme le dit King C. Gillette à la fin de King. Mais la gravité des questions posées par ce théâtre du quotidien n'accable jamais le spectateur, la cruauté n'est jamais exempte de pitié ou de tendresse. C'est que Michel Vinaver a dirigé Gillette France pendant des décennies. S'il a mené une double vie, longtemps dissimulée, c'est qu'il aimait ce travail de direction et s'intéressait à l'entreprise. Il a finalement choisi d'en faire des objets de scène, de représentation et de réflexion, sans être enfermé dans une position d'extériorité didactique. Ce goût n'a rien de cynique ni de masochiste ; l'entreprise lui apparaît comme un champ théâtral où les conflits, les tensions affectives, intellectuelles et libidinales se manifestent comme dans un bouillon de culture. Il l'observe et y collabore en humaniste, fidèle à celui-là même qui tout jeune allait voir Camus à New York, comme s'il fallait qu'il rencontrât Camus pour devenir un homme ${ }^{2}$. Il a finalement unifié sa vie en transmettant par le théâtre et sur scène son expérience du réel.

La force de son théâtre est de rendre la complexité du libéralisme, d'en faire partager l'excitation motrice comme les effets mortifères, de communiquer le plaisir du travail autant que la peine. Loin de l'aplatir et de le simplifier par des stéréotypes, ou de tenir un discours idéologique sur le capitalisme néo-libéral dans un théâtre à thèse, il rend la pâte et la richesse du réel qui déborde les hommes. Certes le dialogue est plein de clichés, d'images toutes faites qui encombrent les cafés du commerce comme les médias : mais ils sont justement mentionnés comme une parole empruntée - aux discours politiques, syndicaux, médiatiques - , qui oblige à penser au lieu d'en empêcher. On le dit ironique, mais cette ironie ne méconnaît jamais la part de fascination pour l'initiative, pour l'énergie, pour l'inventivité et l'ingéniosité, pour les droits de l'individu à accomplir sa tâche et actualiser sa puissance d'agir. Il y a quelque chose de spinoziste dans l'humeur de Vinaver, une positivité que l'ironie n'amuït jamais, une joie du développement, de l'accroissement. Le sens du jeu, l'esprit d'équipe et la jubilation du sport rapprochent la performance d'une entreprise d'autres activités humaines, libres et désintéressées ${ }^{3}$. La bouffonnerie d'Ubu roi n'est pas étrangère à cette jubilation drôlatique et néanmoins critique.

\footnotetext{
${ }^{2}$ Camus aida Michel Vinaver à publier son second roman, L'Objecteur, aux éditions Gallimard (1951).

${ }^{3}$ Ce plaisir sportif et ludique s'entend dans L'Emission de télévision (1988) :

«On a réussi à déjouer pendant quinze ans tous les assauts de la concurrence
} 
C'est le principe du progrès, jadis manifestation la plus extrême de l'optimisme radical et promesse d'un bonheur durable et universel, qui se trouve depuis les pièces des années 80 battu en brèche. Car les choses basculèrent comme si le progrès mythifié engendrait son contraire et favorisait la perte de l'humain après l'avoir pourtant affirmé. La gravité du jeu et le danger du sport sautent alors aux yeux et requièrent les feux de la rampe pour nous éclairer sur le réel auquel nous collaborons.

\section{La dysphorie du renversement : A la renverse et Les Travaux et les jours}

A la renverse répond en 1979 à Par-dessus bord, dont Les Travaux et les jours est le doublet humble et bref. On a entretemps perdu l'illusion de l'illimité de l'expansion au point de penser la nécessité de refonder le capitalisme. La pièce succède à l'expérience d'une grève et d'un conflit social que Vinaver a vécus et traversés comme cadre dirigeant. A la demande de la CFDT, elle fut montée dans l'usine mise en espace par Ernest Pignon-Ernest. Le drame expose la restructuration de l'entreprise familiale Cosson, c'est-à-dire son rachat et la disparition programmée du service après-vente, qui est l'unique lieu de la scène et des échanges. Ce service affecté à l'enregistrement des réparations est aussi un office de doléances car les clients adressent aux standardistes leurs peines et leurs regrets, tandis que celles-ci entre elles partagent inquiétudes ou aspirations. A la fin de la pièce, les objets usés ne seront plus réparés, les employés âgés ne seront plus insérés. Guillermo, le plus jeune et le plus doué d'entre eux, fils d'un républicain espagnol torturé à mort par les franquistes, est limogé sans prime, piégé par l'efficacité qu'opère sur un orphelin la mystique paternaliste de l'entreprise familiale. La violence des échanges dans ce milieu tempéré illustre la déloyale exploitation du sens de l'appartenance et de la hiérarchie. Ne joue plus que la promotion canapé, qui exempte temporairement les jeunes et accortes stagiaires de l'expulsion réservée aux syndicalistes, et plus généralement aux salariés dotés d'une mémoire de l'entreprise qui pourrait la rappeler à l'ordre de son identité fondatrice. La pièce présente l'impitoyable mutation du capitalisme paternaliste à une version néo-libérale où la rentabilité reste seule le critère de l'organisation. Elle lance un avertissement qui voudrait libérer de l'aliénation fondée sur la nostalgie d'un lien fusionnel, voire sacrificiel, telle celle qui

Alors que la plupart étaient dix ou quinze fois plus puissants que nous

Mais nous formions une équipe agile ardente bien soudée ». (Théâtre complet 6, Arles, Actes Sud, p. 152). 
étreint Guillermo. Entré chez Cosson comme on entre en religion, il offre à son nouveau patron sa collection ancienne de moulins à café, comme pour recréer un rapport filial et familial à l'intérieur d'un cadre qui ne l'est plus, ce qu'il est incapable d'assimiler. Mais surtout, la pièce montre la force et la nécessité de ce besoin humain d' «être dans quelque chose »- et non pas de faire quelque chose - par quoi Michel Vinaver définit le travail. C'est ce rapport organique qui participe d'un ordre cosmique et surhumain qu'elle questionne, bien au-delà des échos et résonances criants avec Continental ou Total... multinationales qui exacerbent les symptômes recueillis dans Les Travaux et les Jours. L’ironie des rapports socio-économiques renvoie à l'ironie fondamentale de la condition humaine. C'est un retournement archétypique qu'indexent Les travaux et les Jours et A la renverse. Comme Les travaux et les Jours, renvoie explicitement à Hésiode, je privilégierai cet exemple pour illustrer la dimension philosophique et anthropologique du théâtre de Michel Vinaver.

Le paratexte en est particulièrement fourni et éclairant. Deux passages de l'œuvre d'Hésiode (VIIIème siècle avant Jésus-Christ) encadrent un extrait de la Queste del saint Graal, et un extrait du discours du ministre André Giraud du 20 juin 1979. Le cycle des Travaux et des jours se figure donc dans le bouclage même de cet avant-texte. Mais il n'est pas pure répétition ; la dégradation, la corruption caractérisent le passage de l'âge du blé à l'âge de fer : «Appliquetoi de bon cœur aux travaux convenables pour qu'en sa saison le blé qui fait vivre emplisse les granges. C'est par leurs travaux que les hommes sont riches en troupeaux et en or ; rien qu'en travaillant ils deviennent mille fois plus chers aux Immortels ». Le travail envisagé par Hésiode prend un sens archétypique : il est doté d'une valeur dès lors qu'il assure la vie, et accomplit l'activité humaine sous l'autorité divine. Tant que le travail fait fructifier la terre, le culte des dieux et de l'ordre cosmique est célébré par les semences et les moissons. L’âge du blé qui allie l'effort à l'hommage est béni des dieux. Mais la terre se gâte dès que les hommes oublient le respect des dieux et des pères, sacrifient la valeur d'usage à la valeur d'échange, préfèrent aux fruits les profits. La race du fer et l'âge industriel sont maudits. Autrement dit les hommes occupent une place heureuse tant que le monde naturel vit sous l'autorité d'une transcendance, s'organise en un cosmos hiérarchisé. Leur sort est fragile, leur faiblesse certaine, mais le sens du monde est avéré par les dieux qui suspendent questions et doutes, prémunissent contre l'oubli, l'indifférence et la déréliction. La fin de la tradition archaïque, rurale et religieuse, l'entrée en modernité, en revanche, précipitent soupçons et angoisses, donnent le vertige d'un monde 
déboussolé où les hommes devenus «classe laborieuse » perdent leur place circonscrite certes, mais heureuse : «Et plût au ciel que je n'eusse pas à mon tour à vivre au milieu de ceux de la cinquième race, et que je fusse ou mort plus tôt ou né plus tard. Car c'est maintenant la race du fer. Ils ne cesseront ni le jour de souffrir fatigues et misères, ni la nuit d'être consumés par les dures angoisses que leur enverront les dieux. [...] L'heure viendra où Zeus anéantira à son tour cette race d'hommes périssables : ce sera le moment où ils naîtront avec des tempes blanches. Le père alors ne ressemblera plus à ses fils ni les fils à leurs pères ». Dans l'œuvre d'Hésiode, les dieux se vengent car les hommes dans leur outrecuidance ont cru s'émanciper. Aussi naîtront-ils chenus, vieux avant que d'avoir vécu, usés avant que d'avoir travaillé. Ils ont perdu le sens de la génération, de la filiation et de la transmission: la déliaison, la désolation, l'esseulement leur sont promis à titre de châtiment. Avant de devenir le sujet des négociations affectives (amicales, conjugales, parentales) des personnages de la pièce, dès les exergues de la pièce la fides comme foi spirituelle, respect dû aux anciens et fidélité sentimentale est donc convoquée. Avant même qu'il ait connaissance de la moindre réplique, le lecteur est averti de la dimension " prophétique » de ce théâtre, qui est politique au sens plein du terme. C'est l'avenir de la cité, celui des générations à suivre qui est en question. Celui dont nous portons le poids et la responsabilité. Il faut donc éveiller les spectateurs, «d'abord capturer l'intérêt du public. Ensuite l'amener à un certain état d'instabilité, lui faciliter la voie vers un éventuel basculement, vers un "changement". [...] C'est une manière de déranger l'ordre des choses sans le dénoncer. Toute dénonciation (même chez Brecht) appelle la défense et la contre-offense (contre-offensive ?), l'affrontement et la récupération. Je m'emploie à présenter un monde sans procès (la remarque est de Roland Barthes) mais mû par de petites palpitations qui, à la longue, visent au grand ébranlement ${ }^{4}$. Ce théâtre élabore une stratégie de l'efficacité indirecte : exposer, présenter l'insoutenable légèreté du faire et du manager, interroger le rapport déflagrant de rejet et d'adhésion à la réalité contemporaine, pour faire prendre conscience et rappeler à la société qu'on n'exclut pas impunément l'humain de son cahier des charges. Dans Les Travaux et les jours, le SAV répond aux dégâts matériels comme aux souffrances morales. Le besoin de réparation en passe par la narration. Si aucun ustensile technique ne se répare plus, la moindre parole, elle, se récupère, qu'elle porte sur le fonctionnement des objets ou sur les aspirations des sujets. Mais qui, une fois

\footnotetext{
${ }^{4}$ Etudes théâtrales, I, Lausanne, Editions de L'Aire, 1982, p. 313. La remarque de Barthes est extraite de la préface qu'il a rédigée pour les Coréens (1956).
} 
Cosson absorbé, écoutera les récits de vie qui cherchent une oreille patiente et bienveillante ?

\section{Mythe et histoire}

La dimension pré-historique du mythe n'est jamais éloignée de la mise en intrigue à laquelle se livre Michel Vinaver ${ }^{5}$. Dès 1946, l'exposé «La naissance de la tragédie » atteste son intérêt pour l'épopée et la tragédie antique et mythique. «L'anecdote ou la fable [...] devient de moins en moins accidentelle, de plus en plus essentielle. [...] L'auteur n'a plus la possibilité de choisir. Il faut qu'une fable s'impose à lui, et incarne, et ordonne les formules dont il est le messager. Il suffit de comparer encore une fois l'épopée et la tragédie antique, pour voir combien la structure est insignifiante dans l'une et importante dans l'autre. L'Epopée est le chant du désordre humain. La Tragédie est au contraire la consécration d'un ordre établi » ${ }^{6}$. La fable mythique nourrit donc le théâtre épique de Vinaver, qui raconte autant qu'il expose et parle. King, sur ce double mode narratif et dialogal caractéristique du théâtre contemporain, reconstitue l'épopée de King C. Gillette, descendant d'une famille Gillet chassée de Bergerac, France, par la Saint-Barthélémy, et génial inventeur de la lame de rasoir jetable. Au lieu de retracer l'histoire singulière de l'entrepreneur, sa lancée puis sa ruine des années 1895 à 1929, de louer son ingéniosité technique et spéculative comme la marque d'un génie particulier du capitalisme, Michel Vinaver replace cette histoire exemplaire dans le cadre du mythe. Il en fait à proprement parler une fable. Le symbole des Titans et des Géants qui occupe l'imaginaire de King en dit

${ }^{5}$ Même sur le mode de la dérision, l'inscription d'un imaginaire mythique et archaïque est rappelée par l'invention de sigles comme CIVA dans La demande d'emploi (1973) :

«- Vous savez tout ce que l'Occident puise actuellement dans l'Orient quand il s'agit de trouver les ressources spirituelles nécessaires pour résister aux aspects les plus stérilisants de la société technocratique CIVA C.I.V.A. Communauté Internationale

- De Vacances Agréables?

- Agréables ? Non

- De Vacances aménagées ? De Vacances assistées ? [...]

- De Vacances Animation ». (La demande d'emploi, Paris, L'Arche Editeur, 1973, p. 24-5).

${ }^{6}$ «Vers la naissance de la tragédie », 1946 ou 1947, fonds Michel Vinaver/Archives IMEC. 
long : les Titans combattent, se concurrencent et s'abattent, leur épopée héroïque se conclut tragiquement.

Hésiode toujours, mais cette fois dans la Théogonie, dresse la généalogie des dieux depuis les Titans jusqu'aux Olympiens. Les Titans, dans le mythe archaïque, appartiennent à ce qui n'est pas encore la génération des dieux-enfants, ils sont l'engeance bloquée dans le sein de Gaïa par la puissance d'Eros. Ils sont donc des êtres d'avant le temps. C'est le geste séparateur de Kronos, le cadet, le mieux armé, qui tranche, ordonne, structure, ébranle le temps. Il met en marche l'histoire des dieux Olympiens 7 . Car prévenu par Gaïa qu'il risquait d'être renversé par un de ses enfants, Kronos les dévore tous, sauf Zeus. Lequel à son tour détrônera son père, étendra sa souveraineté sur le monde en fondant la génération des Olympiens... Il en sera puni en la personne de son fils Dionysos, démembré par les Titans jaloux. L'histoire du monde débute donc par le combat sanglant des Titans et des dieux Olympiens, auquel succède la génération des hommes. Quand en effet Zeus foudroie les meurtriers de Dionysos, les vapeurs qui s'élèvent des Titans calcinés se déposent en une suie d'où naîtront les hommes. Issue des retombées de vapeurs, l'âme humaine procède de l'immortalité. Mais le crime titanique est perpétré sur un dieu qui, démembré, disperse la divinité et égrène l'immortalité. L'humanité retient une part de cette suée des Titans et de cette dispersion de Zeus. Les lois d'airain de la concurrence, les restructurations ou ingestions d'entreprises, ne font que réécrire indéfiniment infanticide, parricide, ou « démembrements » selon les mêmes métaphores, répétant le cycle de la vie et de la mort. Pour se venger de Zeus, les Titans tueront et démembreront Dionysos, son fils né de son union avec Perséphone. De l'âge de blé à l'âge de fer, des Titans aux hommes, le mythe hésiodique articule péril de mort, travail et relation à un principe de transcendance. Dans l'histoire de la Modernité, et a fortiori dans le développement néo-libéral, quand ceux qui travaillent se pensent en entrepreneurs, qu'advient-il de la valeur du travail et de sa signification anthropologique? La question se pose à partir de la privation de travail. Dans Les Travaux et les jours, La demande d'emploi, L'émission de télévision, la question du licenciement est omniprésente. Quelle dimension dans cette situation critique le travail revêt-il ?

\footnotetext{
${ }^{7}$ La genèse présente le même paradigme : d'abord le monde créé est unifié, il comprend terre et mer confondues, puis Dieu invente les cieux qui séparent les éléments, font un écran, structurent l'espace. La crise de 1929 dans King s'énonce dans les termes de l'apocalypse : au matin du 24 octobre, il fait nuit et «le ciel se liquéfie ».
} 
La question du travail est toujours anthropologiquement liée à celle des jours à vivre. «C'est la mort qui sert de trait d'union entre le travail et la vie » ${ }^{8}$, comme l'écrit Jan Patocka dans les Essais hérétiques sur la philosophie de l'histoire. Patocka oppose travail et production en articulant ce couple à l'antinomie formée par mythe et histoire. La production dans l'histoire s'oppose au travail dans le mythe : «Le travail conçu dans ce sens apparaît comme un facteur non seulement anhistorique, mais qui s'oppose à l'histoire, qui tend à la retenir au point mort. Mieux qu'aucun autre facteur et pendant plus longtemps, c'est le travail qui a maintenu l'homme dans le cadre du projet de vie de la vie nue, de la vie pour la vie. [...] Loin que ce soit l'histoire qui s'explique à partir du travail, c'est seulement dans l'histoire que le travail conclut avec la production l'alliance qui le rend lui-même dépendant de l'histoire. [...] Seule la production permet de doter le monde humain d'un caractère de pérennité, d'une charpente solide qui vient sous-tendre la molle inconsistance de la reproduction de la vie. Les murailles de la ville, la place du marché, le temple et l'écriture sont des expressions de cette vie affermie » ${ }^{9}$. D'une part le travail permet de subvenir à ses besoins, de détourner le péril de la mort et de survivre comme individu au sens biologique du terme. La production, elle, projette vers l'avenir et donne une sorte d'immortalité aux hommes en tant qu'espèce. Ainsi, la production «affermit» la vie. D'autre part, le travail est anthropogène, il permet une libre réalisation de soi comme personne individuée et autonome. Pour Marx, dès lors qu'il est auto-déterminé, le travail devient un facteur d'émancipation. Il n'y a donc pas seulement dans le travail ce qu'y repère Hannah Arendt, qui l'oppose à l'œuvre et à l'action (praxis) : la peine et la souffrance, l'enfermement dans la survie biologique $^{10}$. Il y a aussi l'affirmation d'un pouvoir, celui d'agir, de prendre conscience de soi et

${ }^{8}$ Essais hérétiques sur la philosophie de l'histoire, 1990, trad. franç. Lagrasse, Verdier, 1999, p. 37.

${ }^{9}$ Idem.

${ }^{10} \mathrm{Cf}$ La condition de l'homme moderne, «Vita activa ». L'activité qui correspond au processus biologique vital signale notre vulnérabilité et condamne au cycle épuisant - au sens plein du terme - production/consommation. En revanche la fabrication de choses qui durent et laissent une trace dans le monde accroît la durée de l'activité humaine. Et c'est seulement de l'action politique qui est à elle-même sa propre finalité que l'on peut attendre véritablement accomplissement et libération. Pour Arendt, le travail ne permet pas de se réaliser comme sujet, puisque les hommes doivent pour se sentir être, accéder à la conscience d'eux-mêmes et se réaliser, être reconnus dans la sphère publique. «La vie affermie » s'oppose à «la vie nue » au sens où la vie réussie engage l'activité libre et la pratique politique. Elle se distingue de la pensée de Marx, pour qui l'expérience du travail comme actualisation de ses forces et réalisation de soi 
de transformer le réel. Son fondement réside pour Patocka en un «libre être-au-monde » qui actualise et oriente le potentiel de l'homme. «Le travail humain présuppose une libre disposition de l'espace et des intervalles temporels ; malgré toute sa monotonie, il n'est pas stéréotypé, mais dirigé vers et par un but. [...] Paradoxalement le travail nous fait éprouver notre liberté ; sa pesanteur dérive de la charge comme trait plus fondamental encore, lié à la vie humaine en général, elle tient au fait que nous ne pouvons pas prendre la vie simplement comme quelque chose d'indifférent, mais qu'il nous faut toujours la «porter», la «mener», nous en porter garants et en répondre ${ }^{11}$. Patocka partage avec Hannah Arendt l'idée selon laquelle le travail rappelle notre faiblesse et notre indigence, mais les relève par l'expédient dont nous disposons pour les surmonter.

L'opposition entre être en vie (la vie nue) et se sentir en vie (la vie affermie) établit la discrimination entre les pauvres invisibles, isolés et fragiles et ceux qui disposent de leur potentiel et de leur force, qu'ils soient de nature sociale, économique et politique. Selon Hannah Arendt, les pauvres sont privés de leur humanité au sens où ils ne disposent pas d'initiative, ne peuvent pas porter de projet. Invisibles, inaudibles, ils se contentent de survivre, sans autre ambition que de manger et se chauffer. Dans notre société, les sans abri, sans domicile fixe, sans emploi de même survivent, dans les marges de la société. Ils sont niés dans leur valeur sociale, jusqu'à perdre confiance en soi et estime de soi. Si l'on emprunte à Axel Honneth les catégories de La Lutte pour la reconnaissance, la perte de l'estime de soi touche les individus qui ne peuvent plus contribuer par leurs pratiques, par un travail formellement organisé à la vie commune; pour les chômeurs «en fin de droits », s'ajoute à cette marginalisation la perte du respect de soi comme membre d'une communauté d'égaux en droits ; enfin, la dissolution de la confiance en soi comme personne digne d'affection atteint le père de famille qui ne peut plus pourvoir à l'entretien du foyer ni garantir l'éducation de ses enfants ${ }^{12}$.

est contrariée par la logique du capitalisme. Désaliéné, le travail reste la condition nécessaire pour élaborer un rapport à soi satisfaisant et il prend une valeur éthique.

${ }^{11}$ J. Patocka, Essais hérétiques sur la philosophie de l'histoire, p. 35.

${ }^{12} \mathrm{Cf}$ A. Honneth, La Lutte pour la reconnaissance, en particulier le chapitre 5 (trad.franç. P. Rusch, Paris, Editions du Cerf, 2000). Pour Axel Honneth, le principe de la reconnaissance est un principe normatif qui gît au cœur du social, traversé par la dynamique du conflit et de la concurrence. 


\section{La vie nue}

La demande d'emploi (1973) comme L'émission de télévision (1988) dénoncent les effets criminogènes de la logique de la rentabilité comme finalité. La demande d'emploi exemplifie dans les années 70 une économie théâtrale resserrée dans une trentaine de scénettes autour d'un trio familial (père - chômeur - , mère et fille) et d'un recruteur. Michel Vinaver s'essaie à l'abolition de toute chronologie et à un montage inspiré par les variations Goldberg ou Diabelli. L'empiètement de la sphère privée et de la sphère professionnelle, les effets paradoxaux induits par le libéralisme s'enchevêtrent : la libération des mœurs et de la sexualité amène le thème de l'avortement dans La demande d'emploi (avant la loi Weil c'est un thème important du théâtre du quotidien; il succède à celui du couple à trois dans Les travaux et les jours) tandis que le libéralisme économique condamne le «travailleur» potentiel à de multiples contorsions acrobatiques. Il s'agit en effet d'incarner à la fois l'unique, l'authentique et l'irremplaçable collaborateur et nonobstant quelqu'un d'adaptable, de flexible, de « formatable ». A cette double contrainte paradoxale, s'ajoute un handicap supplémentaire pour les plus de cinquante ans. La respectueuse appellation de senior n'est qu'idéologique dès lors qu'elle les exclut du développement social d'une culture jeuniste. Ce sont des individus rendus superflus par leur âge, que seules peuvent accueillir, aux mêmes fins de rentabilité, des émissions racoleuses et pathétisantes comme celle de Vincent Bonnemalle dans L'émission de télévision. La métaphore de l'excédent attire à elle dangereusement celle du déchet ou de l'excrément. «Des déchets il faut des déchets pourquoi est-ce qu'il n'y aurait pas des déchets ? Vingt-trois ans pour devenir un déchet », demande Fage dans La demande d'emploi, quand l'angoisse et la honte l'ont convaincu de la légitimité de sa mise au rebut ${ }^{13}$. Le déchet est le devenir de l'objet réifié, l'expulsion et le cassage ceux de la réification. Cette déshumanisation pousse à sa dernière extrêmité la déconsidération de soi, qui exemplifie ce que Bourdieu nomme dans La Misère du monde la «misère de position ${ }^{14}$ : la misère de condition renvoie à l'indigence matérielle effective alors que la misère de position se vit. C'est le vécu subjectif de qui occupe une place inférieure, obscure et s'éprouve comme si inutile, si extérieur à l'espace public et aux activités collectives qu'il ne mérite plus de se respecter, de s'estimer, de s'appréhender comme sujet affectif, juridique et social. L'émission de télévision met en compétition deux chômeurs de plus de

\footnotetext{
${ }^{13}$ La Demande d'emploi, p. 60.

${ }^{14}$ Bourdieu, La Misère du monde, Paris, Seuil, 1993.
} 
cinquante ans, dits «de longue durée » et sans espoir de «retour à l'emploi ». Ils sont en concurrence non seulement sur le marché du travail mais sur un plateau de télévision pour une émission de télé-réalité conforme aux attentes émotionnelles d'une heure de grande écoute: «De la façon dont nous concevons cette émission il y aura un certain nombre d'itinéraires tragiques aussi variés que possible qui seront reliés par un thème commun le chômage et l'effet destructeur que produit celui-ci sur le tissu familial mais aussi sur la personnalité profonde de l'individu ${ }^{15}$. Le comble du cynisme est atteint lorsque ceux à qui la société, en les privant d'une inscription professionnelle et sociale, retire toute visibilité et toute projection possible vers un avenir sont exhibés comme des bêtes de foire suréclairées sous les projecteurs. Le malheur se vend bien, et il «paie ». L'émission de télévision suspecte les collusions d'intérêt, le manque d'étanchéité entre le monde politique, le monde judiciaire et le monde médiatique. De l'entrepreneur à la pigiste en passant par le juge, tous courent à la « réussite » de la gloire et sacrifient à la vanité d'être connus par les médias. Tandis que le réalisateur Bonnemalle dîne chez le Garde des Sceaux, « un petit juge d'instruction pour sa publicité personnelle jette en prison à quelques jours de l'émission celui que Vincent Bonnemalle avait choisi pour porter l'espoir à ces centaines de milliers de malheureux que sont les chômeurs ayant pour seul crime d'avoir plus de cinquante ans ${ }^{16}$. La médiatisation compassionnelle bat son plein, selon les règles de figuration et de stylisation commandées par la chaîne ${ }^{17}$. Du moins le ferait-elle, si la loi de la concurrence n'impliquait pas de se débarrasser du concurrent, lui aussi superflu: de l'abattage à l'abattement, il n'y a qu'un pas $^{18}$. Cette surexposition médiatique ne compense pas la passivation forcée ni l'exclusion, elle en fait de l'argent. On fait jouer aux «chômeurs de longue durée » leur rôle de passifs et de patients, qui nie pour la vie toute possibilité de réalisation de soi et de responsabilisation. On ne peut participer à la société du spectacle qu'en consentant à sa mutilation de meurtri: "Au plan des médias il ne peut occuper qu'une place celle de la victime c'est en quelque sorte

${ }^{15}$ L'émission de télévision, Théâtre Complet 6, p. 131.

${ }^{16}$ Ibid., p. 283.

${ }^{17}$ Cf M. Revault d'Allonnes, L'Homme compassionnel, Paris, Seuil, 2008, pp. 38-45. Sur l'exhibitionnisme télévisuel, voir Alain Ehrenberg, L'Individu incertain, Paris, Calmann-Lévy, 1995.

${ }^{18}$ Pas que franchit le protagoniste du Couperet, qui exécute méthodiquement chacun de ses concurrents. Cette logique de l'exécution et de l'élimination des superflus est suggérée par le film de Nicolas Klotz, La Question humaine (2007). 
structurel $»^{19}$. Jouer ce rôle consistera à résumer le quotidien d'un demandeur d'emploi, à récapituler les symptômes de sa dégradation morale, que l'on peut décrypter aussi comme ceux d'une « pathologie sociale » :

-Tu envoyais tes quinze lettres par jour

Chaque semaine une relance

L’attente des réponses

- Il n'y avait pas de réponses

- Ou c'était non

- Et progressivement l'affolement

- Parfaitement oui on peut le dire Le doute qui se transforme en certitude Tu ne vaux plus un pet

La certitude qui vous dévaste

Le deuil de soi

- Je le voyais se dissoudre à vue d'œil je n'y pouvais rien $\gg^{20}$

Le deuil de soi impliqué par le chômage est à coup sûr une pathologie sociale courante. Les pathologies sociales sont des pathologies de la reconnaissance, puisque la reconnaissance accorde une valeur sociale et qu'on lutte pour elle à condition de le pouvoir. Axel Honneth reprend à son compte les analyses des perturbations du processus de l'individuation ${ }^{21}$. L'individu en «demande d'emploi » ne fait plus que l'expérience de sa faiblesse et de son impuissance, il ne peut plus développer aucun rapport à soi satisfaisant, à moins de s'imaginer autre qu'il n'est. Par exemple, la stratégie de résistance à la «dissolution de soi » passe pour Blache par une fiction valorisante et universalisante. Il n'est plus un limogé mais un naufragé, il ne vit plus dans une société qui lui dénie une place mais sur une île déserte. Cette affabulation en donnant une portée universelle à son état lui permet de survivre en héros. Le mythe vient au secours de la vie:

${ }^{19}$ L'émission de télévision, p. 289.

${ }^{20}$ Ibid., p. 157.

${ }^{21} \mathrm{Cf}$ A. Honneth, La Société du mépris, Vers une nouvelle Théorie critique, «les pathologies du social », Paris, La découverte/Poche, p. 39 - 100. Selon Emile Durkheim, l'individuation désigne le processus d'affranchissement par rapport à la tradition et aux contraintes normalisantes qui permet à l'individu de se révéler et de se choisir à l'intérieur de la société comme un de ses membres. 


\begin{abstract}
«Robinson sur son île ça a été le déclic dans mon désoeuvrement forcé je m'étais mis à ranger les livres par ordre alphabétique

Robinson Crusö̈ un de mes vieux livres d'enfance dans sa reliure rouge

Je l'ai feuilleté il faut croire que ça m'a travaillé pendant mon sommeil le lendemain matin au réveil $\mathrm{j}$ 'ai dit à ma femme [...]

Il m'a dit je suis Robinson sur son île je ne suis pas un vieux je suis un naufragé qui a réussi tu entends Caroline réussi

A gagner une île déserte

Un naufragé ça organise sa survie même si l'attente est longue une voile finira par apparaître

Entretemps il faut gérer la situation dans la pleine acceptation de toutes ses contraintes

Devant l'étendu vide demeurer actif attentif scruter l'horizon prêt à envoyer les signaux ${ }^{22}$
\end{abstract}

Or la voile et les signaux qui apparaîtront seront néfastes, et la pièce s'achève par une mise à mort. L'utopie est de croire possible de s'émanciper du travail ou de la privation de travail et de la souffrance, de croire pouvoir échapper à la part nocturne de l'existence humaine. La mort rôde toujours, dans le geste de «couper » qui concerne le montage technique de l'émission comme dans le rapport tragique entre individu et société : «Entre la société et l'individu tombe la hache $»^{23}$. Le nombre de films de fiction récents mettant en scène l'enjeu de vie et de mort dans la question de l'emploi est impressionnant, du Couperet de Costa Gavras (2005) à Sauf le respect que je vous dois de Fabienne Godet (2006). Sans oublier les documentaires comme Ils ne mouraient pas tous mais tous étaient frappés, où Christophe Dejours explicite les pathologies sociales du chômage comme du travail précarisé, où les sujets se vivent comme les illégitimes survivants de licenciements collectifs ${ }^{24}$.

\title{
Liquidité et déliaison
}

${ }^{22}$ L'émission de télévision, p. 160.

23 Ibid., p. 276.

${ }^{24}$ Cf C. Dejours, Souffrance en France, La banalisation de l'injustice sociale,. 1998, éditions du Seuil, 1998. 
Ces temps de naufrage social s'accompagnent de l'affaiblissement des liens communautaires et de la désolidarisation. Si phonétiquement et sémantiquement solide est proche de solidaire, la défection de ces liens rapproche par antinomie liquide et liquidé, dissous et désolé. La palette est large, de la désaffiliation à l'atomisation individuelle des collègues qui ne col-laborent plus. La revendication de l'autonomie individuelle accouche diaboliquement d'exigences drastiques et contradictoires, de plus de discipline et de plus d'instabilité, qui défait toute forme de solidarité sociale. L'individuation, qui accomplissait la liberté du sujet social raisonnable et responsable, s'achève en individualisation égocentriste qui signifie l'isolement dans un réseau de contacts anonymes ${ }^{25}$. La différenciation aboutit à l'indifférence. Dans $L a$ demande d'emploi, la désaffiliation aboutit paradoxalement à un discours libertaire antiavortement... par lequel la fille-mère justifie le "bébé jetable" : elle veut «savoir ce que c’est qu'accoucher nourrir le mener jusqu'à ses premiers pas après on le refilera il y a des quantités de couples qui cherchent un enfant $»^{26}$. Le fossé entre père et fille se creuse d'un malentendu sur l'épreuve de la subjectivation :

«Regardez ma fille Nathalie elle réclame la révolution elle à seize ans épuisée déjà de faire ce qu'elle veut et quand je dis faire elle ne fait pas elle est charriée par ce qui survient quand je dis veux elle ne veut rien elle est sujette à des petits vouloirs seulement ces petits vouloirs ça vous lamine une vie familiale il n'y a pas de communauté possible [...] il n'y a pas trente six mille choses nouvelles dans le monde alors il faudra bien revenir en arrière ha la révolution ça dit bien ce que ça veut dire ça tourne ça tourne et tout revient au même ${ }^{27}$

La passivité, l'instabilité des jeunes des années 70 augurent-elles l'identité fluide postmoderne ? Les caprices et pulsions nés du hasard ou de la séduction confondus avec le libre arbitre libèrent Nathalie de l'obéissance aux parents et de l'inscription généalogique mais ne la

${ }^{25}$ Contacts et non plus communication, qui plus est souvent dans une sociabilité virtuelle.

${ }^{26}$ La Demande d'emploi, p. 31.

27 Ibid., p. 57. King assortissait l'euphorie de la jetabilité, signe d'émancipation de la peur de manquer et de mourir, à celle de la traçabilité, signe de la maîtrise et du contrôle du réel par les procédures d'enregistrement rationnel. Au commencement était la raison, puis vint son instrumentalisation. La lame Gillette était jetable, c'est désormais celui qui l'utilise qui l'est. 
libèrent pas pour quelque chose. Surtout pas pour assumer l'à venir d'une manière inédite, puisque de l'à venir elle se débarrassera. Pour un être abandonné à ses pulsions, flottant et désarçonné, il n'y a plus qu'à croire à la révolution sans même savoir envisager l'amplitude sémantique du mot. Nathalie incarne-t-elle une forme de personnalité nouvellement apparue qui se caractériserait par l'effritement des rôles traditionnels, la multiplication des identités, une pluralisation intrapsychique de sujets liquéfiés? Elle revendique une identité fragmentée, hédoniste, répondant aux impulsions de l'instant : «Je veux enfin j'essaie de vivre d'une façon déconnectée on fait une chose pleinement une autre pleinement on n'essaie pas de relier sinon» ${ }^{28}$. La déliaison, l'éclatement de la durée en instants isolés tournent le dos au concept de responsabilité qui engage la continuité temporelle et se fonde sur le concept de subjectivité.

La fluidification de l'identité postmoderne est un avatar de l'image de la liquéfaction répandue dans les essais de sociologie et d'économie contemporains, des flux tendus à la circulation des liquidités, des liquidations judiciaires à La société liquide. Le concept de Zygmunt Bauman qualifie la société obsédée par le changement, la flexibilité, pratiquant le culte de l'éphémère et, partant, ouverte aux pratiques d'interchangeabilité, de jetabilité et d'exclusion. L'amour liquide, de la fragilité des liens entre les hommes traite de la façon dont les êtres humains tentent de négocier leurs relations, amoureuses, professionnelles, sociales, dans une économie de surproduction, de compétition, et de désinstitutionnalisation. Toutes les sphères, privée et publique, affective et professionnelle communiquent par capillarité selon les lois du capitalisme en réseau. C'est cette vie affective et intime parasitée par le libéralisme que décrit Michel Houellebecq dans Extension du domaine de la lutte. L'homme policé, civilisé est sacrifié sur l'autel de son propre dieu. Dans tous les cas, la technique mise au service de la maîtrise et du contrôle a raison des pratiques intersubjectives, des relations humaines et de la communication éthique. De la lame à l'homme jetable, fût-il encore bébé ou à peine mort, il n'y a plus de différence.

\section{L'Ordinaire ou la fin de l'exception humaine (1982)}

${ }^{28}$ La Demande d'emploi, p. 35. 
L'Ordinaire résulte de l'adaptation d'un fait divers, l'accident de l'avion qui transportait une équipe de sportifs tombé dans les Andes. Les rescapés, de moins en moins nombreux, survécurent en se nourrissant de la chair de leurs camarades morts. Michel Vinaver substitue à l'équipe de footballeurs l'équipe de cadres d'une entreprise multinationale florissante pour dénoncer l'anthropophagie du capitalisme. La mutation d'un code à un autre occupe la pièce et en fait la gravité. La métaphore de l'ogre convient d'abord à la multinationale Housies qui a dévoré ses concurrents et qui pratique l'élimination interne des rivaux encombrants : parce qu'il faut bien manger, les collaborateurs participent à cette dévoration sans fin. Ed raconte à Sue l'épopée Housies:

Ed

Au départ ils étaient trois Tenenbaum Green et Lamb

Green et Lamb ont éliminé Tenenbaum le fondateur celui qui avait eu l'idée

Ensuite ça a été le duel à mort entre Green et Lamb Green était le technicien Lamb était le vendeur

Moi j'étais un homme de Green son assistant l'homme à tout faire son plus proche confident

Green a pensé se débarrasser de Lamb en le laissant s'embrarquer dans le projet Europe Green a cru que Lamb allait s'enliser en Europe Lamb a ouvert l'Europe il a éliminé Green

Sue

C'est comme ça que ça se passe ?

[...] Et vous êtes resté ?

Ed

Il fallait bien manger ${ }^{29}$

Le parricide fait toujours recette, la faim légitime toute collaboration. Avant cet ultime "passage », cette dernière «mutation », la pièce rend l'excitation du projet d'expansion et aussi l'obsession quasi pathologique du PDG qui d'ailleurs meurt non pas de faim, mais d'une crise cardiaque : sans doute ne peut-il survivre à l'échec de son implantation en Amérique latine. Plutôt que d'être implanté au Chili, il est bel et bien planté dans la cordillère des Andes ! L'excitation l'emporte sur l'exigence, le politiquement juste résonne avec l'économiquement juteux. Avant de mourir, Bob a certifié avoir acheté le monopole par le soutien démagogique accordé à Pinochet : «Nous implantons une couronne de Housies autour de chaque

${ }^{29}$ L'Ordinaire, TC 5, p. 189-190. 
agglomération de plus de dix mille habitants [...] moyennant quoi Pinochet nous consent des avantages fiscaux très importants vous avez vu dans le rapport de Larry franchise d'impôt sur le revenu pendant cinq ans exclusion de tous les concurrents potentiels $»^{30}$. Le politiquement correct justifie de soutenir la dictature au nom du bien du peuple pourvu que le triomphe de Housies soit « couronné » par la couronne de baraquements qui « casse[nt] la baraque ») :

Le coup de l'Europe il y a vingt-cinq ans a été génial

Mais le coup de l'Amérique latine est encore beaucoup plus énorme un coup d'une intelligence confondante

Parce que c'est un coup politiquement juste

Bob avait cette intelligence cet instinct qu'on n'acquiert pas à Harvard Avec ça on casse la baraque

La couronne de Housies pour remplacer les assemblages de tôles et de cartons autour des principaux ensembles urbains

Les cinq mètres carrés par personne entièrement équipés qui redonnent à l'homme sa dignité pour le prix d'un mètre carré bâti en matériaux traditionnels

Dans la conjoncture qu'ils traversent c'est pile ce dont ces généraux ont besoin ${ }^{31}$

Le capitalisme a préparé le cannibalisme. Recommandé par la loi sacrée de la survie biologique, il devient naturellement un acte naturel d'animalité. Avant de mourir de gangrène, Pat suggère «les morts ça doit pouvoir se manger». Quand Sue apporte la première tranche de viande humaine séchée au soleil, Bess s'insurge :

Bess

Ce qu'elle a fait maintenant

Bob je vais me sentir mal dis quelque chose

Dieu est là-haut qui nous regarde Il attend qu tu prennes position

Toi qui détestes être mis devant le fait accompli

Il y a des choses qui sont sacrées

Pat

$\mathrm{La} \mathrm{vie}^{32}$

Cet acte naturel d'animalité signe simultanément la fin de cette exception humaine sur laquelle la phénoménologie avait mis l'accent. Dans la pensée de Husserl, le terme " esprit" réfère à ce qui est irréductible ontologiquement à l'identité biologique, psychologique et sociale

${ }^{30}$ Ibid., p. 40.

${ }^{31}$ Ibid., p. 160.

${ }^{32}$ Ibid. , p. 68. 
de l'être humain. Or dans un essai au titre choc, La fin de l'exception humaine ${ }^{33}$, Jean-Marie Schaeffer souligne la continuité entre le monde naturel non humain et l'espèce humaine biologique. Cette contiguïté de l'inhumain et de l'humain serait avérée par les récents travaux sur le cerveau, la psychologie expérimentale et le développement des sciences sociales. La thèse de l'exception humaine est alors réduite à une vision $d u$ monde illusoire et mystificatrice. Dans la pièce L'Ordinaire, aucune morale ne résiste à l'évidence animale : «la vie est sacrée ». Ce laboratoire théâtral d'une expérience sociale exploite le fait divers réel pour mettre à l'épreuve une idée de l'Homme. Hélas, dévoyé qu'il est dans l'histoire contemporaine des rapports humains, aucun humanisme n'est plus crédible. Tout est, de jure, consommé, dès lors que tout est de facto consommable.

\section{Théâtre du réel}

Le théâtre de Vinaver ne représente pas l'ordinaire, mais présente l'épaisseur poisseuse, désordonnée et contingente du réel pour le constituer en quotidien. « La banalité quotidienne n'a jamais cessé de [1]'intéresser. La matière de la vie journalière dans son état le plus indifférencié, le plus confus, les plus neutre ». Inspirée par l'esthétique dadaïste ou cubiste du montage et du collage, l'écriture de Michel Vinaver avance non pas linéairement, mais par rassemblage, irradiation. Les répliques ne s'enchaînent pas mais s'enchevêtrent, entrent en collision et produisent des éclats de sens, qui sont autant d'éclairs de sens. En renonçant aux agencements logiques, à l'ordre communicationnel de la pertinence et de la relation, « des déflagrations [qui] produisent du sens », disait l'auteur à propos de la mise en scène de Par-dessus Bord ${ }^{34}$. «Des choses brutes (micro-événements, gestes, paroles) sont amassées ${ }^{35}$ de sorte que leur juxtaposition abrupte permet d'établir des connexions, d'avancer dans la connaissance, en forçant un accès vers le réel. Ce théâtre diagnostic offre une expérience heuristique, accouche de la vérité contemporaine des relations humaines. Fait comprendre sans surplomber, c'est-à-dire prendre et composer à mesure que les répliques se succèdent. Embranchements, clignotements renvoient à

\footnotetext{
${ }^{33}$ J.M. Schaeffer, La fin de l'exception humaine, Paris, Gallimard, 2007.

34 Mise en scène de Christian Schiaretti produite par le Théâtre National Populaire de Villeurbanne, puis montrée à Paris au Théâtre de la Colline en mai-juin 2008. Si cette mise en scène peut satisfaire l'auteur, c'est qu'elle conserve et fait signifier la fragmentation, l'éclatement, l'entrelacs de voix et de sujets (trente lieux et soixante personnages).

${ }^{35}$ Ecrits sur le théâtre 2, L'Arche, 1998, p. 90.
} 
un imaginaire dynamique et immanent. La non-hiérarchisation des propos et des personnages, le désordre accidentel et contingent des enclenchements restitue la problématicité du monde, son opacité et sa complexité, à la fois angoissante et excitante. Ce théâtre de la perplexité «sert à remuer nos idées, à les mettre en mouvement, au risque du déséquilibre ${ }^{36}$.

Or cette mission est plus urgente que jamais. Du temps conflictuel officiel des Coréens (1957) au temps brumeux et clandestin de 11 septembre 2001, le théâtre de Vinaver illustre un théâtre des idées et de l'énergie cinétique: «Et si le théâtre des idées était un théâtre qui remue les idées du spectateur? Qui ne laisse pas en place nos idées, qui les met en branle ? ${ }^{37} \mathrm{La}$ distanciation éclairante et désaliénante passe par l'entrelacs et le tressage qui empêche tout abandon à la mimesis et toute crédulité fascinée. Ce tissage textuel remplace la distanciation épique qui convenait à un monde idéologiquement binaire. Il en tient lieu dans le chaos où nous sommes: «L'effet d'éloignement est ce qui permet de se dégager de la glu dans laquelle cherchent à nous maintenir, mentalement, les pouvoirs de ce monde. Mais Brecht cadrait sur un monde volontairement simplifié. Aujourd'hui, [..] face aux systèmes oppressifs qui s'enchevêtrent pour mieux nous tenir, il ne se dégage pas clairement de vérité qui vaille le combat. Au conflit succède la bouillie. Alors la distanciation, qui plus que jamais importe dans le travail théâtral, ne s'obtient plus par l'acte de brandir, de désigner à bout de bras; elle s'intériorise, elle est constante dans l'écriture même, et doit l'être dans le jeu des acteurs. Elle réside, au niveau moléculaire de théâtre, dans le refus de ce qui est attendu » ${ }^{38}$. L'univers théâtral de Michel Vinaver, loin du jugement, de l'autorité surplombante et condescendante, favorise le discernement, fidèle aux injonctions de L'Exception et la règle: «Sous le familier découvrez l'insolite. Sous le quotidien décelez l'inexplicable ».

La choralité sert au mieux cette vocation heuristique : aucune voix dominante ne résiste au choc de la polyphonie. Dans 11 Septembre 2001, « la forme se rapproche de celle des cantates et des oratorios, se composant d'airs (à une, deux ou trois voix), de parties chorales (qui, dans la version française, restent dans la langue originale), et de récitatifs pris en charge par un «journaliste », fonction qui peut

\footnotetext{
${ }^{36}$ Edwy Plenel, «Au vif, L'affaire Vinaver », Le Monde 2, 30 avril 2005.

${ }^{37}$ Ecrits sur le théâtre 2..

${ }^{38}$ Théâtre public, $\mathrm{n}^{\circ}$ 39, 1981.
} 
faire penser à celle de l'évangéliste dans les Passions de J.S. Bach ${ }^{39}$. L'irréductibilité tragique du conflit est annoncée d'emblée. L'information force à la lamentation, puisqu'aucun discours ne peut ni prétendre couvrir l'autre de sa tonitruante arrogance ni pourtant renoncer à le vouloir. Pour un occidental moderne, le sacrifice des terroristes s'appuie sur un mépris du corps, de la femme et de la vie non seulement étranger à ses propres valeurs, mais en outre disqualifié par la puissance de déni et d'aliénation qu'il recouvre ${ }^{40}$. Pourtant l'obsession impérialiste n'est pas plus convaincante, soit que la collectivité économique, par la voix du chœur, n'annonce immédiatement par prétérition des licenciements, soit que le président Bush incite compulsivement à la consommation :

Chœur

On Wall Street

Times are Difficult

Jobs Have to be Cut

Yet More Companies are Reluctant

To Dismiss People Immediately

For Fear of Seeming Heartless

Bush

Il est vital

De continuer à consommer

Chœur

Keep Desires Afloat

Bush

${ }^{39} 11$ Septembre 2001, note liminaire. La langue originale est l'anglo-américain, l'adaptation française a été rédigée ensuite par l'auteur.

${ }^{40}$ Atta rédigeant son testament cinq ans avant cet attentat kamikhaze «ne savait pas qu'il n'y aurait pas de corps », comme dit le journaliste :

Je ne veux pas qu'une femme enceinte ou une personne mal propre

S'approche pour me faire des adieux

Je veux qu'aucune femme ne vienne sur ma tombe pendant mes funérailles ou à quelque autre occasion par la suite». 11 Septembre 2001, Paris, L'Arche, 2002, p. 53. 
De continuer à acheter

Pour prémunir l'économie contre la menace

D'un effondrement

Chœur

For This is War Prepare

To Suffer Hardships

Consent Sacrifices ${ }^{41}$

En écho, Bush et Ben Laden en appellent à leur dieu, et cette échoïté identifie absurdement les adversaires et confond les irréconciliables :

Bush

Bonjour sur mes ordres l'armée des Etats-Unis a commencé des frappes

Ben Laden

Voici l'Amérique frappée par Dieu tout-puissant en un de ses organes vitaux

Bush

Nous sommes soutenus dans cette opération par la volonté collective du monde entier

Ben Laden

De sorte que ses plus grands édifices sont pulvérisés par la grâce de Dieu à qui va notre gratitude

[...] Le camp des croyants

Bush

Et nous n'échouerons pas

Ben Laden

Et le camp des mécréants

Bush

${ }^{41} 11$ Septembre 2001, p. 53-55. 


\section{La paix et la liberté l'emporteront}

Ben Laden

Que Dieu nous protège

Bush

Que Dieu nous bénisse $»^{42}$

Dans Power Inferno, Jean Baudrillard établit le lien indissoluble entre les deux discours et les deux pouvoirs infernaux. Il appréhende le terrorisme comme une «Contre-puissance vitale aux prises avec la puissance de mort du système. Puissance de défi à une mondialité totalement soluble dans la circulation et dans l'échange. [...] Le système lui-même, par l'extension spéculative de tous les échanges, la forme aléatoire et virtuelle qu'il impose partout, les flux tendus, les capitaux, la mobilité et l'accélération forcée, fait régner un principe général d'incertitude que le terrorisme ne fait que traduire en insécurité totale. [...] Il parachève l'orgie de puissance, de libération, de flux, de calcul, dont les Twin Towers étaient l'incarnation ${ }^{43}$. Expansion et exagération de la prétention à la vérité et à la puissance, la mondialisation excède l'impérialisme. En cela elle partage la logique d'anéantissement du terrorisme, elle disloque le monde en interdisant la différence et l'altérité, en les intégrant dans le tout indifférencié. L'esthétique de l'éclat et du passage, du fragment et du zapping coïncide avec une dénonciation tonale et rythmique de cette logique de mort dont nous sommes acteurs, passeurs, et à terme victimes. Elle donne la mesure de l'atomisation et de la scission tout en revendiquant, sur le mode de la résistance, le droit au reste.

Finalement l'œuvre de Michel Vinaver, qu'elle s'accomplisse en un théâtre de chambre ou en un théâtre de la cité, questionne la relation à l'autre, les conditions de possibilité d'une entente et d'un dialogue. Elle interroge aussi les circonstances nécessaires d'une culture morale «qui confère aux intéressés, méprisés ou exclus, d'articuler leurs expériences dans l'espace public démocratique au lieu de les mettre en acte dans le cadre des contre-cultures de la

${ }^{42}$ Ibid., p. 61-69.

${ }^{43}$ J. Baudrillard, Power Inferno, Requiem pour les Twin Towers, Paris, Galilée, 2002, p. 36-37. 
violence $»^{44}$.

Marie-Hélène Boblet

Université Pari III - Sorbonne nouvelle

${ }^{44}$ A. Honneth, La Société du mépris, « la dynamique du mépris », p. 202. 